\title{
OFFSET-CONTINUATION-TRAJECTORY (OCT) DATA REGULARIZATION TO A 2D OCEAN BOTTOM NODE (OBN): FULL-WAVE INVERSION APPLICATION
}

\author{
Alexandre Camargo (D), José Ribeiro (iD, Tiago A. Coimbra (iD, \\ Gustavo Barroso iD, and Martin Tygel(iD)
}

\begin{abstract}
Estimating attributes for a better understanding of subsurface geological structures is essential in many seismic exploration and production stages. As a powerful seismic inversion tool, Full Waveform Inversion (FWI) has been widely used to estimate many of such attributes with high resolution. In many cases, however, real acquisitions cannot provide the quantity and density of traces required for optimal inversion results. Consequently, schemes to produce new traces by interpolation/extrapolation of neighboring available traces play a crucial role. Another challenge of using a real dataset in an inversion process is the presence of certain types of noises, which are inherent to the seismic acquisition process and can be treated, generally, by the use of a band-pass filter. This paper uses the Offset-Continuation-Trajectory (OCT) stacking method to generate new traces and include them in an Ocean Bottom Node (OBN) acquisition for non-deeper water. Furthermore, we also use the OCT method for dataset enhancement. Next, we test the FWI on the enhanced dataset and analyze the results. Our approach is applied to a synthetic dataset obtained from an OBN acquisition carried out on the 2D Marmousi model as a proofof-concept. Several additional traces, simulated by the OCT procedure, have been included. Despite the recognized complexities of the Marmousi model, we have obtained very encouraging results.
\end{abstract}

Keywords: regularization, full waveform inversion, signal-to-noise enhancement.

RESUMO. A estimação de atributos físicos é essencial em muitos estágios da sísmica de exploração. Como uma poderosa ferramenta de inversão sísmica, a inversão da forma de onda completa (FWI, do inglês full waveform inversion) tem sido amplamente utilizada para estimar tais parâmetros com alta resolução. Contudo, em muitos casos, uma aquisição real pode não ser capaz de fornecer uma quantidade de traços exigidos para a obtenção de bons resultados. Assim, métodos que produzem novos traços por interpolação/extrapolação a partir de traços vizinhos desempenham um papel fundamental no processo de inversão sísmica. Outra dificuldade é a presença de certos ruídos, inerentes ao processo de aquisição do dado sísmico, geralmente, tratados usando certos tipos de filtros, como um passa banda. Neste trabalho, usamos o método denominado trajetória de continuação do afastameto (OCT, do inglês offset-continuation trajectory) para regularizar traços e incluí-los no sistema de aquisição escolhido, neste caso, do tipo OBN (do inglês, ocean bottom node) para superfícies rasas. Além disso, o método aumenta a relação sinal-ruído. Aplicamos FWI nos resultados da regularização para analisar se o dado regularizado obtém melhorias na inversão. Como prova de conceito, nossa abordagem é aplicada para um dado sintético 2D produzido por uma aquisição OBN usando o modelo de velocidade Marmousi, reconhecido por apresentar certas complexidades geológicas. Os resultados obtidos confirmam que utilizar o método OCT traz benefícios tanto no processo da inversão quanto no processamento do dado sísmico.

Palavras-chave: regularização, inversão da forma de onda completa, melhoria da relação sinal-ruído. 


\section{INTRODUCTION}

Full Waveform Inversion (FWI) is a high-resolution seismic imaging method based on the complete wavefield to obtain subsurface medium parameters, e.g., the wavepropagation velocity (see, e.g., Lailly, 1983; Tarantola, 1984). The difference between the amplitudes observed and the synthetic events of interest serves to perform an inversion process of updating the medium parameters (Virieux and Operto, 2009; Fichtner, 2011). Nowadays, both industrials and academics use and research several approaches and applications of the FWI (e.g., Crase et al., 1990; Abreo et al., 2018; Huang et al., 2021). However, the method still faces many challenges related to inverse-scattering imaging due to seismic data processing, e.g., irregular acquisition geometry, poorly illumination, and datasets with a low signalto-noise ratio (SNR) (Jouno et al., 2019; Wang et al., 2020).

As mentioned by Bleistein et al. (2001), the inversion methods, in the general case, have a problematic issue that is the coverage limiting. I.e., the dataset information has not sampled sufficiently, and therefore, the presence of spatial aliasing is caused. Thus, to improve the seismic inversion process to obtain better results, it is necessary to increase the number of shots and receivers. In other words, the seismic dataset must be sampled in a dense grid. Quite often, however, acquisition configurations are unable to provide full coverage. Several factors explain this poor coverage, including physical limitations (e.g., finite-length cables (streamers), environmental and topographic difficulties), instrumental failures, and the expensive cost of acquiring a dense data sample. As such, interpolation/extrapolation methods that can simulate new traces (unrecorded traces) from neighboring ones (recorded traces) are always in demand (Gülünay, 2002; Trad et al., 2002; Fomel, 2007). In particular, such schemes can enable cost-reduced acquisitions as fewer traces need to be recorded. As described in Fomel (2003) and (Coimbra et al., 2012, 2016) and based on the Common-Reflection-Surface (CRS) parameters (Jäger et al., 2001), the Offset-Continuation-Trajectory (OCT) stacking is able to simulate, from a given CommonOffset (CO) section determined for a given half-offset, the corresponding continued $\mathrm{CO}$ section that corresponds to a new, user-selected, different half-offset. In this way, the OCT method can be used as a tracesimulation tool to provide trace-enhanced datasets better suitable for seismic imaging.

A question that naturally arises and we intend to answer in this work is whether an enhanced dataset with constructed traces, via stacking process, is still suitable for FWI application. However, we must remember that such a procedure depends not only on the tracereconstruction precision but also on the robustness of the $\mathrm{FWI}$ procedure, as the FWI is based on local optimization, i.e., it depends on an appropriate starting point (Nocedal and Wright, 1999).
In order to address the interpolation and regularization problem in an Ocean Bottom Node (OBN) acquisition geometry (see, e.g., Gaiser, 2016), in this work in non-deeper water, we consider a synthetic dataset computed with the 2D Marmousi velocity model (Versteeg, 1994), where Figure 1 illustrates a typical OBN acquisition. First, we use the OCT method for regularization (increasing fold), increasing the SNR. Besides, we apply FWI in the regularized dataset. We compare the FWI results obtained with the original full-trace datasets and the corresponding ones obtained by the OCT method. For our numerical experiments, FWI results showed a better resolution using the OCT regularized dataset. Further natural steps can encourage the consideration of the procedure on real datasets to make it practically feasible.

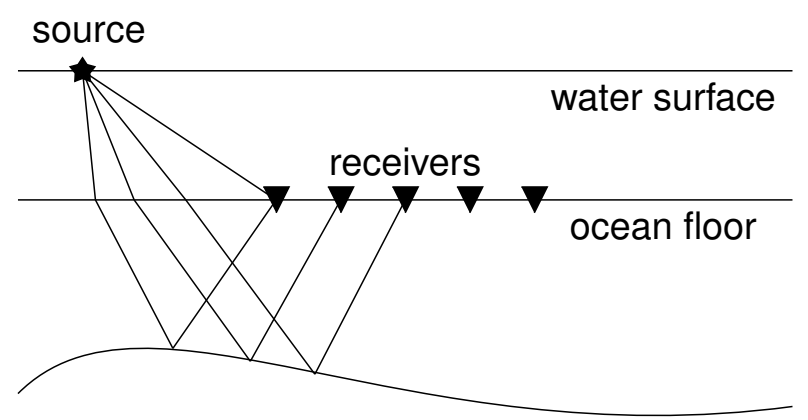

Figure 1. Illustration of an OBN acquisition. The star represents the source and the triangles represent the receivers on the ocean floor.

\section{THEORY AND METHOD}

We briefly describe and expose the main definitions of the OCT and FWI algorithms used in this work. A diagram of both methods is provided in Figure 2. As our main focus is to test the proposed combination of OCT and FWI to a specific dataset, we refrain from providing the technical exposition of these algorithms, referring the interested reader to adequate publications available in the literature (see, e.g., Virieux and Operto, 2009; Coimbra et al., 2016).

\section{Offset Continuation Trajectory Stacking}

The Offset Continuation Operation (OCO) described in Deregowski and Rocca (1981) and more recently in Coimbra et al. (2012) is an operator that transforms traces on a recorded $\mathrm{CO}$ section, defined by a given half-offset, into the corresponding (unrecorded) $\mathrm{CO}$ traces of a different, continued half-offset. Besides that, Coimbra et al. (2016) highlighted the great potential for improvement on data stacking in the time domain using the OCO operator. The stacking is composed of an OCO trajectory, namely, in their work, by OCT stacking. In this way, non-existent traces that belong to a CO section can be simulated by recorded traces 


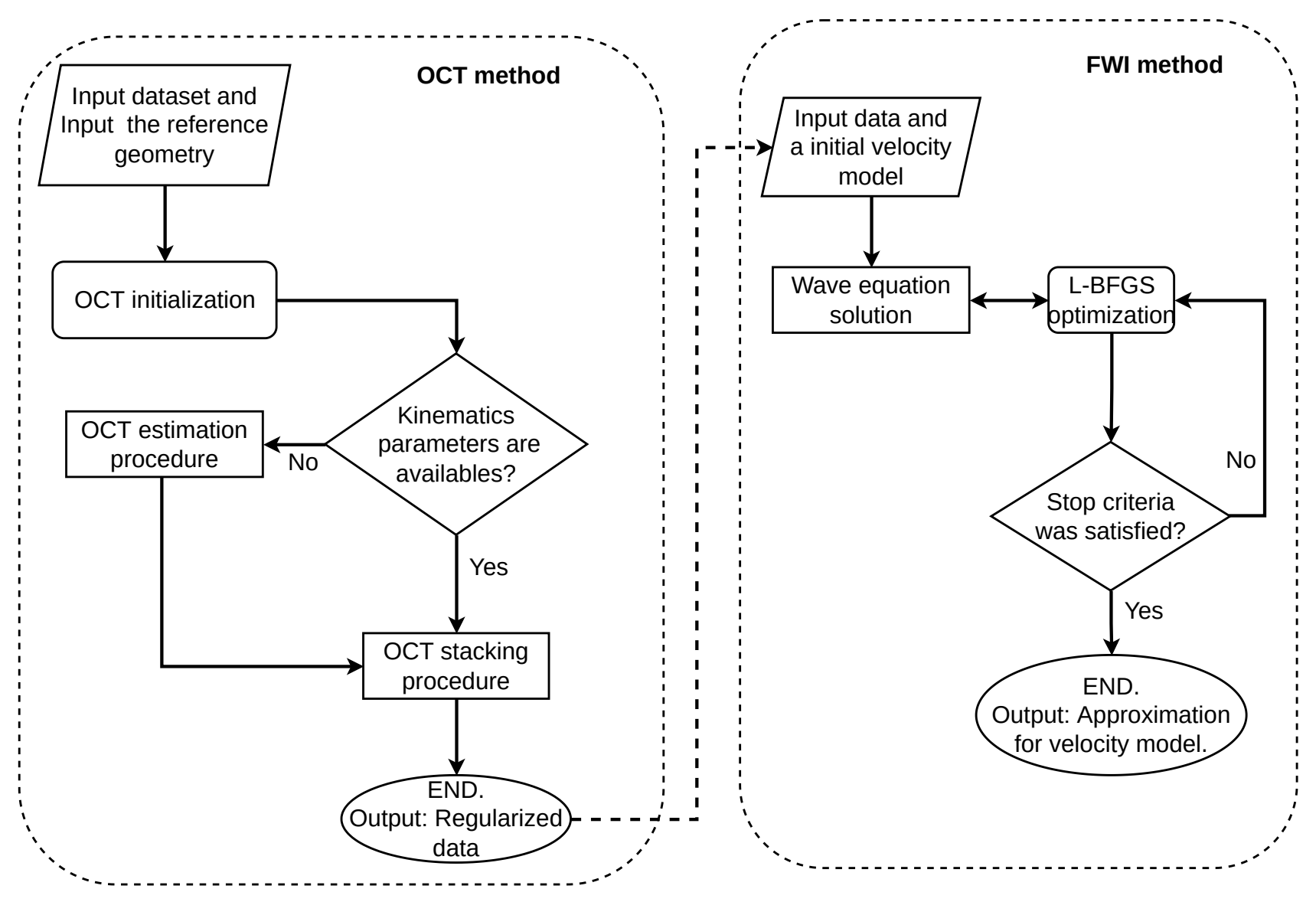

Figure 2. Flowchart of OCT-FWI approach.

that belong to a $\mathrm{CO}$ section of a neighboring half-offset. The process requires only two parameters: local slopes and stacking velocity. The estimation of these parameters also uses information from the input data. However, it requires careful analysis in its processing. Thus, the method stacks the data along predicted traveltime curves that approximate the true Common-ReflectionPoint (CRP) with appropriate parameters, i.e., the OCO trajectory approximates the CRP trajectory.

\section{Full Waveform Inversion Application}

For a given acquisition configuration, FWI is carried out as an iterative process, which consists of solving a forward (see, e.g., Sommerfeld, 1949; Bleistein, 1984; Carcione et al., 2002) and an inverse problem (Fichtner, 2011; Leeuwen and Herrmann, 2016). An initial model for the attributes desired to be inverted is supposed to be provided. Usually, seismic tomography methods build the initial models (Prieux et al., 2012). In our case, a single attribute of acoustic velocity model is considered.

With the help of that initial model, the forward problem consists of computing all the shot records available in the original dataset. That computation is carried out by solving the acoustic wave equation under the use of classical Finite-Difference Modeling detailed in (Kelly et al., 1976; Holberg, 1987).

For inverse problem, the obtained shot records are compared with their corresponding observed ones, giving rise to a misfit value that is iteratively minimized in a least-squares sense using the Limited-memory Broyden-Fletcher-Goldfarb-Shanno (L-BFGS) method proposed by Liu and Nocedal (1989) and used in many seismic applications such as inversion (Asnaashari et al., 2013; Wang et al., 2013) and migration (Wu et al., 2015; Souza and Schleicher, 2017). Since FWI is a local optimization method, a downward direction is desirable. In our case, we use the gradient method computed by the adjoint state method (see, e.g., Plessix, 2006; Fichtner, 2011). Regarding step length employed in the optimization algorithm, we decide to use a strong Wolfe's condition, a set of two inequalities that guarantees a sufficient decrease in the function and curvature, providing an upper and lower bound on the admissible step length values. The process is iterated until a given stop criterion is reached, provided by the maximum iteration number or the optimization tolerances. The optimization algorithm is based on algorithms detailed in Nocedal and Wright (1999). As indicated above, we are here only concerned with actual applications, so that a deeper technical description of the algorithms of finite difference method (FDM) and FWI theory is out of the scope of this paper. For more specific details concerning the algorithms used in this work, we recommend the excellent tutorial Virieux and Operto (2009). 


\section{NUMERICAL EXPERIMENTS}

The following numerical experiments are divided into two applications. First, we analyze the ability of the OCT method for noise handling, i.e., leading to an increase of the signal-to-noise ratio. Second, we apply the method to estimate new traces to increase coverage. Finally, we apply the FWI method to analyze how the previous processing impact the inversion of the acoustic propagation velocity.

As depicted in Figure 1, we consider the idealized situation of an OBN seismic acquisition performed on subsurface Marmousi velocity model as illustrated in Figure 3. Under such conditions, an observed dataset, called the Reference dataset, is simulated. As described by the second column of Table 1, the Reference dataset consists of shot records that correspond to 321 surface point sources and 601 receivers at a planar sea bottom surface located at $0.5 \mathrm{~km}$ depth. The shot records are simulated as the solutions of the acoustic wave equation, being obtained by the FDM for a centered scheme of second-order in time and fourthorder in space (Alford et al., 1974; Husted et al., 2004). Besides that, as the computational domain is finite, to mitigate boundaries reflections, we use the absorbing boundary conditions based on Kosloff and Kosloff (1986). A Ricker wavelet gives the point source with a peak frequency of $20 \mathrm{~Hz}$. The FDM parameters, which satisfy the numerical criteria of stability and dispersion, are $\Delta x=\Delta z=0.01 \mathrm{~km}, \Delta t=1 \mathrm{~ms}$ for a maximum recording time $T=3 \mathrm{~s}$.

Besides the Reference dataset, we also consider two additional datasets, designated by Noisy and Bit datasets, with acquisition parameters provided by the third and fourth columns of Table 1, respectively. Thus, the Reference data would be the ideal data we would like to have, while Noisy and Bit datasets are the ones that will pass through the seismic processing. To facilitate the understanding of OCT applications, we split into two sections the results obtained, namely: Signalto-Noise Enhancement and Increasing Fold Coverage.

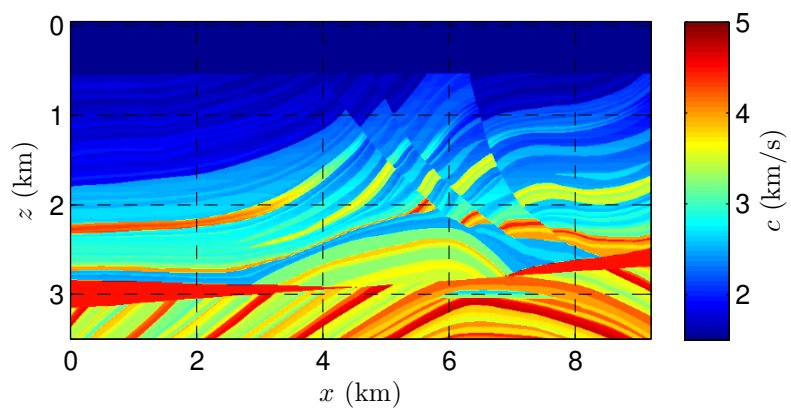

Figure 3. Marmousi depth-velocity model.

Figures 4 (a), 4 (b), and 4 (c) show the Reference dataset in three shot coordinates (in $\mathrm{km}$ ), i.e., $\mathbf{x}_{s_{(\mathrm{a})}}=$ $(1,0), \mathbf{x}_{s_{(\mathrm{b})}}=(4,0)$, and $\mathbf{x}_{s_{(\mathrm{c})}}=(6,0)$, respectively. This data will be our target to be achieved. For the same shot positions, Figure 5 depicts the common-shot gathers of the Noisy dataset. the Bit dataset are shown in
Table 1. : Dataset acquisition parameters for Reference, Noisy, and Bit datasets.

\begin{tabular}{|l|c|c|c|}
\hline \multirow{2}{*}{ Parameters } & \multicolumn{3}{|c|}{ Experiments } \\
\cline { 2 - 4 } & Reference & Noisy data & Bit data \\
\hline $\begin{array}{l}\text { Number } \\
\text { of shots }\end{array}$ & 321 & 321 & 321 \\
\hline $\begin{array}{l}\text { Number } \\
\text { of receivers }\end{array}$ & 601 & 601 & 61 \\
\hline $\begin{array}{l}\text { Shot } \\
\text { separation } \\
\text { (km) }\end{array}$ & 0.025 & 0.025 & 0.025 \\
\hline $\begin{array}{l}\text { Receiver } \\
\text { separation } \\
\text { (km) }\end{array}$ & 0.01 & 0.01 & 0.1 \\
\hline $\begin{array}{l}\text { Maximum } \\
\text { offset (km) }\end{array}$ & 7 & 7 & 7 \\
\hline
\end{tabular}

Figure 6. All common-shot sections have the same amplitude scale, and the noise factors were generated by applying a signal-to-noise set to ten using Seismic Unix (SU) software (see, e.g., Forel et al., 2005). To avoid the use of the raw noise in the FWI method, a bandpass filter implemented by SU for the frequency band 5 to $65 \mathrm{~Hz}$ was used. We applied the same procedure for the Bit dataset, but we have also performed one experiment with noise-free data.

As previously indicated, the Reference dataset will be used to compare regularized Noisy and Bit datasets (i.e., after applying the OCT method).

\section{Signal-to-Noise Enhancement}

As the first application, let us show how the OCT stacking method can be a powerful tool in data processing for increasing the signal-to-noise ratio. This experiment did not obtain new traveltimes because the Reference and Noisy data have the same acquisition geometry. However, the process is the same to approximate new traveltimes.

As described in Coimbra et al. (2016), the OCT trace simulation makes use of the kinematic parameters, as in the CRS procedure, slope in midpoint direction, and velocity-average information. Such parameters are directly extracted from the original dataset, being initialized for the zero-offset (ZO) stacked dataset and then iteratively updated for increasing half-offsets. Figures 7 and 8 show the initial (ZO) midpoint slopes and the velocity-average information. These two parameters used in OCT stacking are estimated using a given dataset on the reference geometry, i.e., 321 shots and 601 receivers, with the maximum half-offset aperture 
(a)

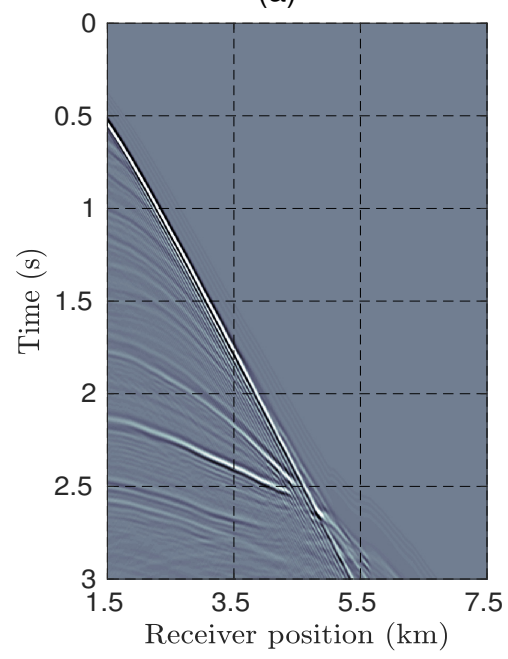

(b)

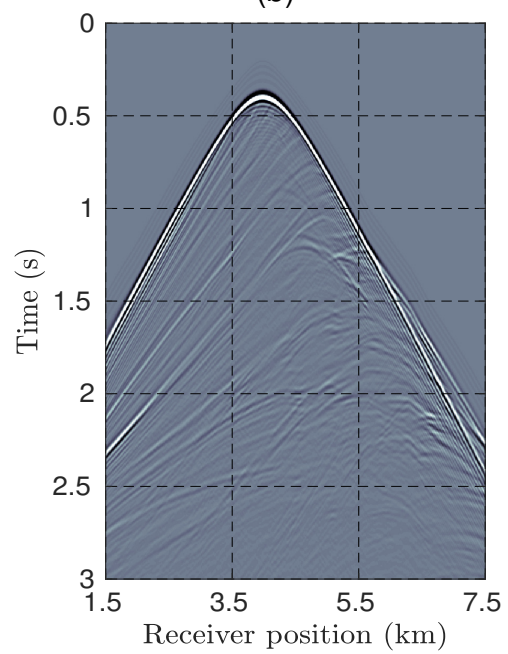

(c)

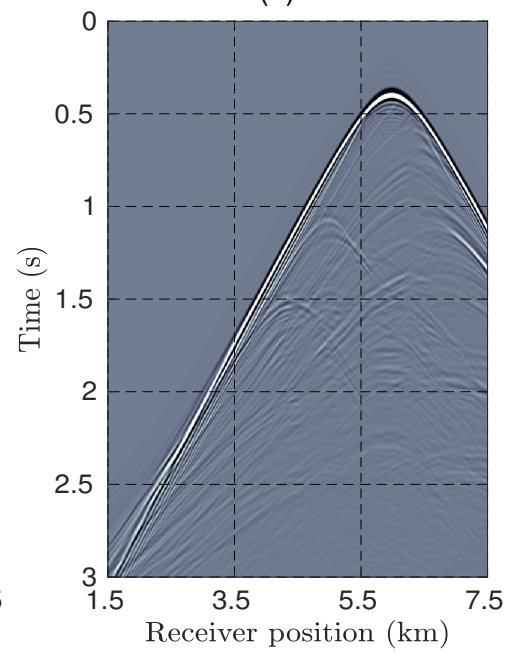

Figure 4. Reference dataset for the shot position: (a) $1 \mathrm{~km}$; (b) $4 \mathrm{~km}$; (c) $6 \mathrm{~km}$.

(a)

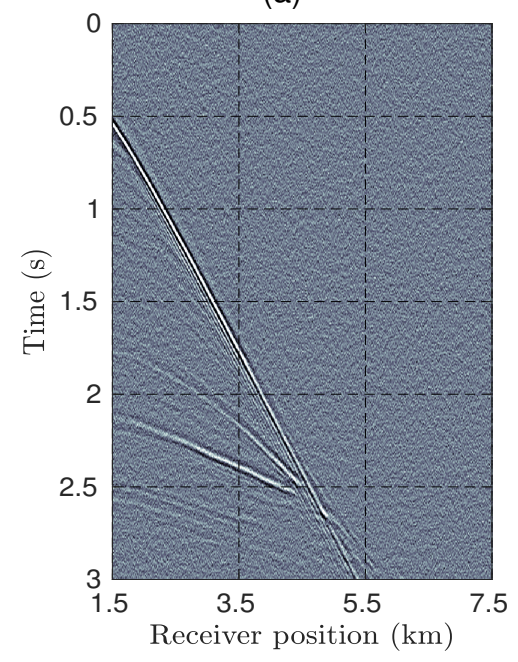

(b)

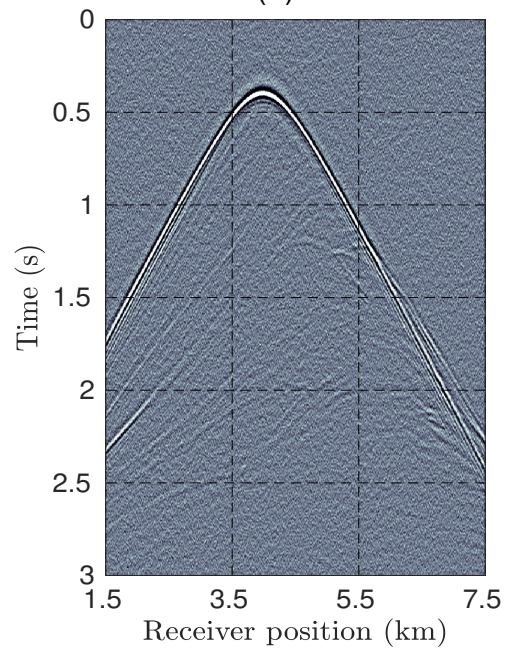

(c)

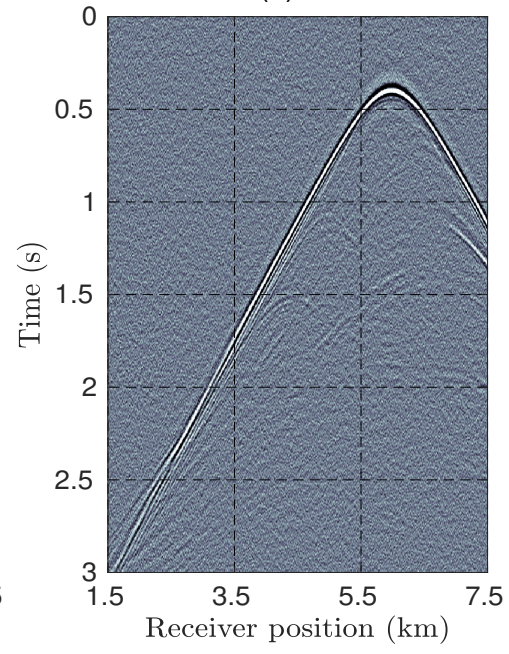

Figure 5. Noisy dataset for the shot position: (a) $1 \mathrm{~km}$; (b) $4 \mathrm{~km}$; (c) $6 \mathrm{~km}$.

(a)

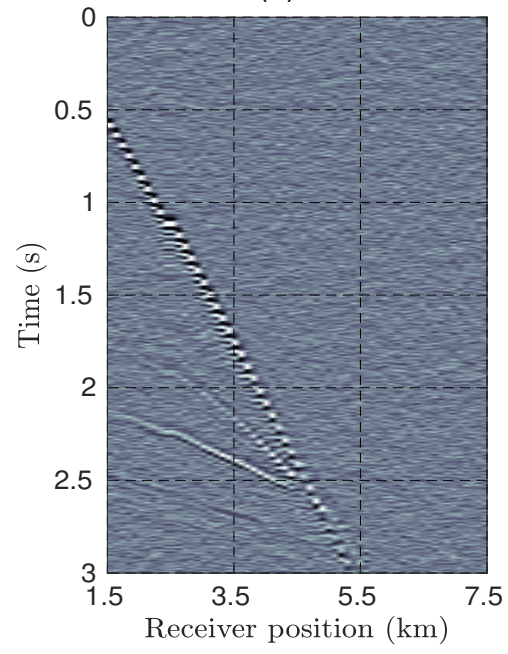

(b)

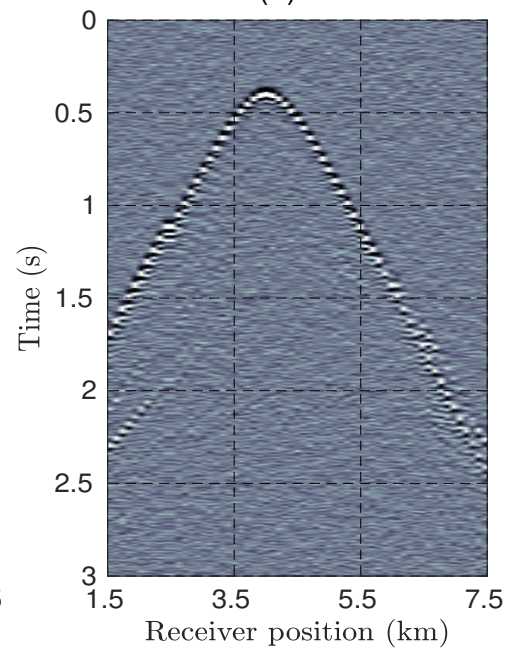

(c)

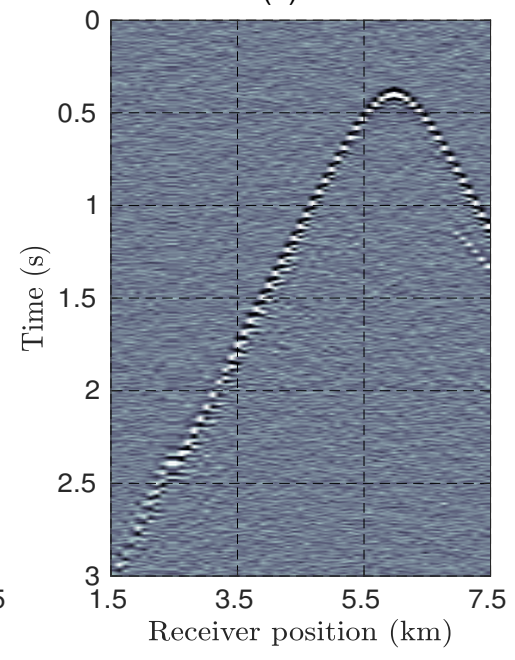

Figure 6. Bit dataset for the shot position: (a) $1 \mathrm{~km}$; (b) $4 \mathrm{~km}$; (c) $6 \mathrm{~km}$. 
set to $0.5 \mathrm{~km}$ and maximum midpoint aperture set to $0.15 \mathrm{~km}$. In order to stack the dataset, the half-offset and midpoint aperture were set to $0.05 \mathrm{~km}$. However, a more careful analysis would provide more accurate parameters.

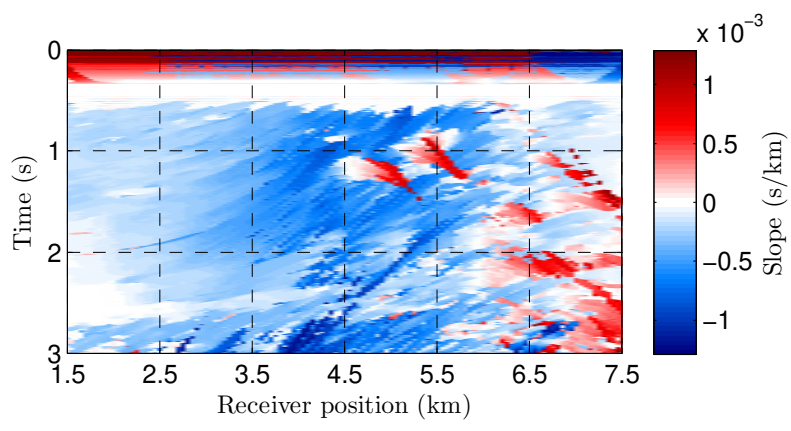

Figure 7. The initial OCT slope parameter in ZO gather estimated from Noisy dataset.

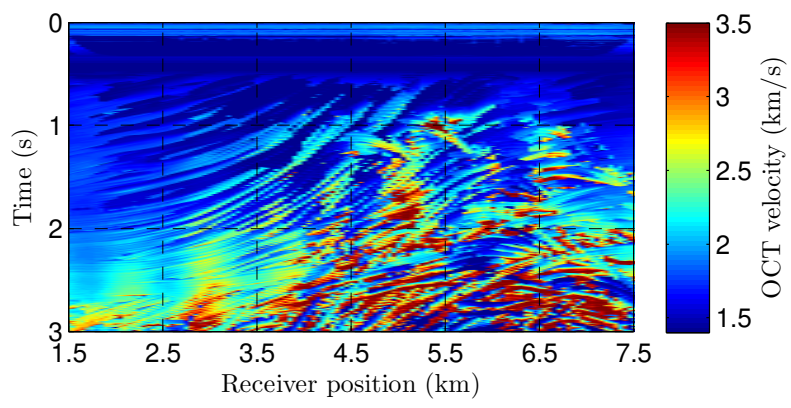

Figure 8. The OCT velocity parameter in ZO gather estimated from Noisy dataset.

Regularized common-shot gathers obtained by OCT in the Noisy dataset are shown in Figure 9 for three shots positions. Based on this experiment, the signalto-noise enhancements can be observed in the Noisy dataset after the applications of the OCT stacking procedure. All main reflection events are preserved, and only the information near direct wave events presents weak accuracy.

Comparing Figure 10 (a) and Figure 10 (c), we can see the robustness of the regularization provided by the OCT method in the ZO section of the the Noisy dataset. Figure 10 (b) shows the ideal ZO section for this experiment. Based on Figures 10 (a)-(c), the regularized Noisy dataset is much closer to Reference than (original) Noisy dataset. Also, after the application of OCT method, we observed that it is possible to see events that were harmed before by the noise. Furthermore, the amplitude scale is the same, except for the Noisy (original) dataset that was chosen appropriately to get a better visualization of the amplitude scale.

Finally, in order to compare locally the improvement introduced by the OCT method, we compared two trace positions for the shot position located at $4 \mathrm{~km}$. Figures 11 (a), 11 (c) show the traces from the Noisy and Reference datasets, where it is observed the difficulty to visualize reflection events, except for the direct wave event. However, after the use of the OCT method, we obtain cleaner data with a significant increase in the signal-to-noise ratio, as we can see in Figures 11 (b), 11 (d), where we can observe the events with reasonable accuracy close to the Reference dataset.

\section{Increasing Fold Coverage}

For the second application using the OCT method, we are now concerned with building approximate traveltimes that were not recorded in the field. To show this application, we have performed two experiments designated by case $A$ and case $B$. Case A considers the estimated parameters (slope and stacking velocity) from Noisy data. Therefore, stacking procedure was performed with half offset and midpoint apertures set to $0.05 \mathrm{~km}$. On the other hand, case B performs a new parameter estimation using the Bit dataset, but noisefree. The apertures used were set to $0.5 \mathrm{~km}$ for half offset and $0.15 \mathrm{~km}$ for midpoint. Once the demanded parameters are obtained, we follow stacking data using an aperture of $0.05 \mathrm{~km}$ for both coordinates.

\section{Analysis: Case $A$}

In case A, the OCT is applied with slope and stacking velocity parameters obtained from the previous section of the Signal-to-Noise Enhancement. In this way, we assume that the parameters used by the OCT method are the best possible, since they were calculated using all the available data. The results of the regularized Bit data (or abbreviated by Bit-A) are shown in Figures 12 (a)-(c). We confirm that the OCT method can increase coverage without adding spurious events since the amplitude scale is the same as the Reference dataset. Futhermore, the fold coverage has been increased about ten times more than the original Bit dataset. They are economically attractive for OBN acquisitions that typically demand more expensive technology. Figures 13 (a),(b), in the ZO sections, confirm that the OCT method achieves improvements apparent to the Regularized dataset if compared with the original Bit dataset, respectively.

\section{Analysis: Case B}

In this case, the OCT is applied to the noise-free Bit dataset, where firstly the estimation of the slope and stacking velocity parameters only uses the Bit data information. The half-offset and midpoint apertures used were $0.5 \mathrm{~km}$ and $0.15 \mathrm{~km}$, respectively. Then, we carry out the stacking using $0.05 \mathrm{~km}$ for apertures for both measurements and obtain the data regularized in the geometry of the Reference dataset, that is, with greater coverage. The regularized Bit-B dataset is illustrated in Figures 14 (a), 14 (b), and 14 (c) with a suitable approximation of the events compared to the Reference data. For another perspective, the ZO section depicted in Figure 15 shows that the solution obtained by the 
(a)

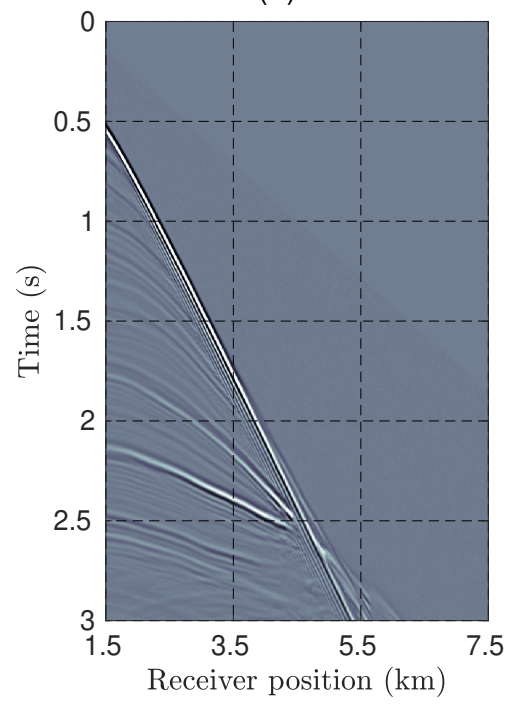

(b)

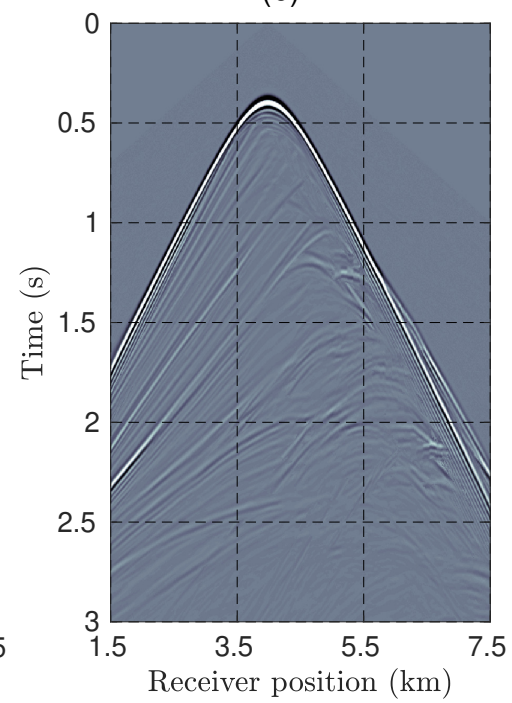

(c)

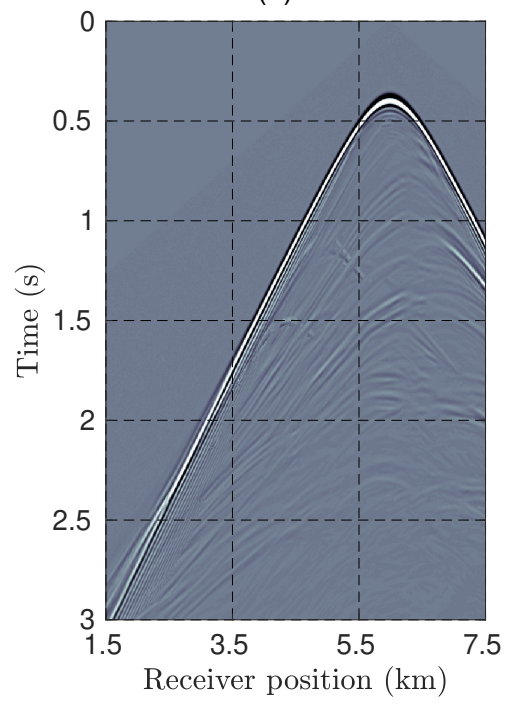

Figure 9. Regularized Noisy dataset by OCT method for the shot position: (a) $1 \mathrm{~km}$; (b) $4 \mathrm{~km}$; (c) $6 \mathrm{~km}$.

(a)

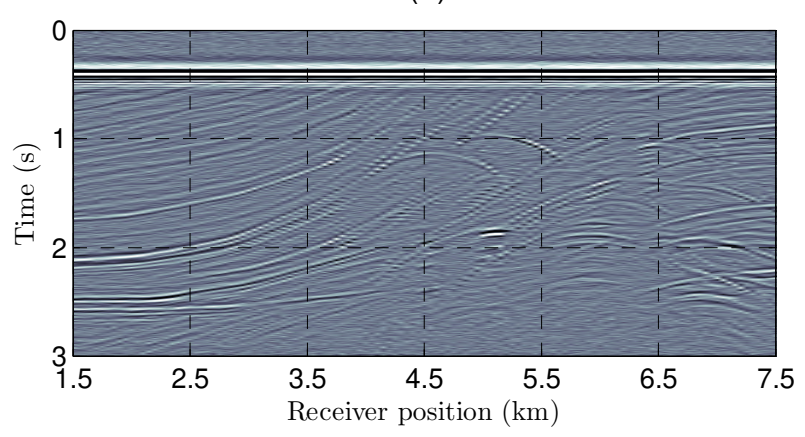

(b)

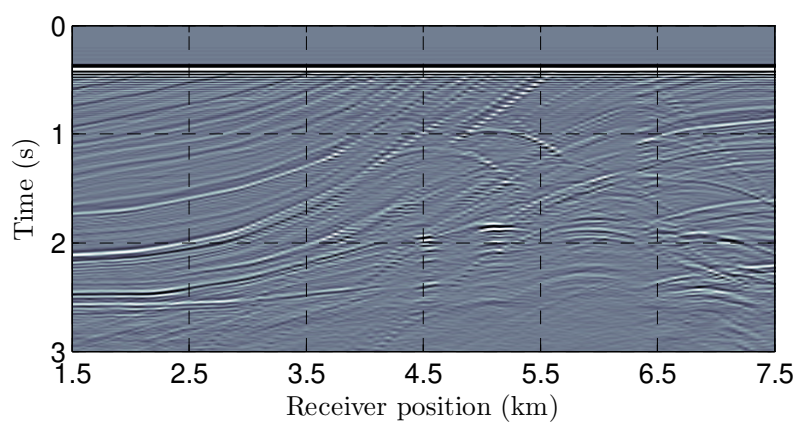

(c)

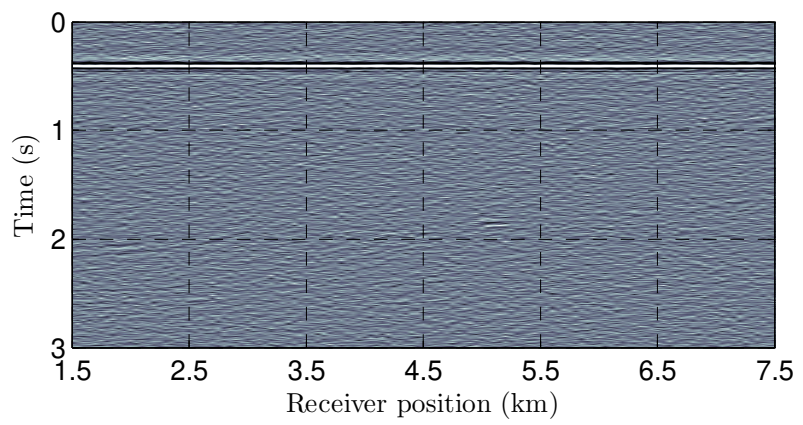

Figure 10. ZO gather for: (a) Regularized Noisy dataset; (b) Reference dataset; (c) Noisy dataset.
OCT method can simulate new features from the existing neighboring features with good accuracy.

As a more profound analysis was not carried out regarding the apertures used in the estimation of the OCT parameters, we also noticed that the regularization of Case B presents certain events very close to the smoother direct wave events when compared to Case A. Thus, we cannot forget to mention that the success of the regularization depends on the use of good slope and stacking velocity parameters.

Finally, let us compare two trace positions at the same position analyzed in the Signal-to-Noise section for both cases to finish this analysis. Nevertheless, this time, we pay attention to the $4.990 \mathrm{~km}$ position that was not recorded in the Bit dataset's acquisition geometry. As we can see, Figures 16 (a), (c) show a good coherence of events even under the presence of noise affecting the signal for Case A. On the other hand, without the noise effects (Case B), the approximation of reflection events for the regularized dataset are with reasonable accuracy, as depicted in Figures 16 (b) and (d). However, as noted earlier, case B has certain smoother events due to the parameters used in the OCT method.

\section{Results of Inversion}

We now focus on the FWI application method to our given datasets. As already mentioned, our FWI computation uses the quasi-Newton optimization method LBFGS proposed by Liu and Nocedal (1989) with optimal tolerance of $\varepsilon=10^{-05}$. All the implementation was done in GPU. In this work, we use the conventional FWI method, which can be found in Tarantola (1984); Virieux and Operto (2009). However, recent improvements to the method can already be found in Qu et al. (2019) and Wellington et al. (2019). The experiments were performed using Amazon Web Service EC2 instances (N. Virginia) cloud computing with virtual machine ubuntu p3.2xlarge Intel Xeon 2.30 GHz, RAM 60GB, 8 CPUs 
(a)

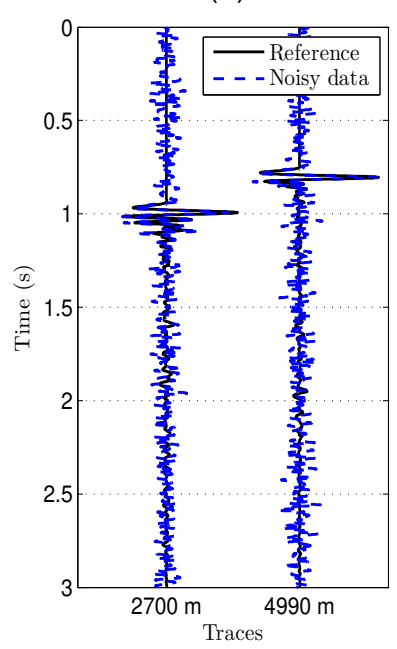

(c)

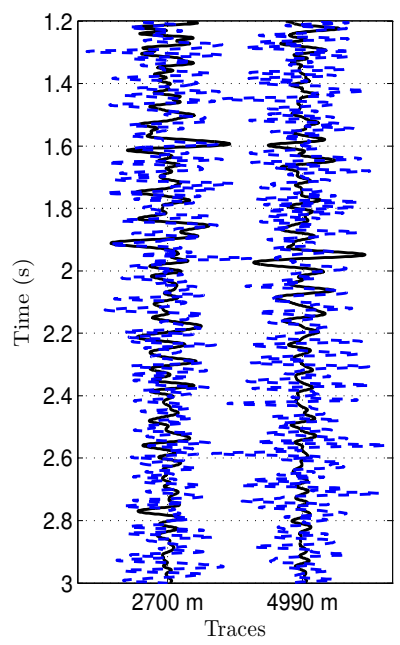

(b)

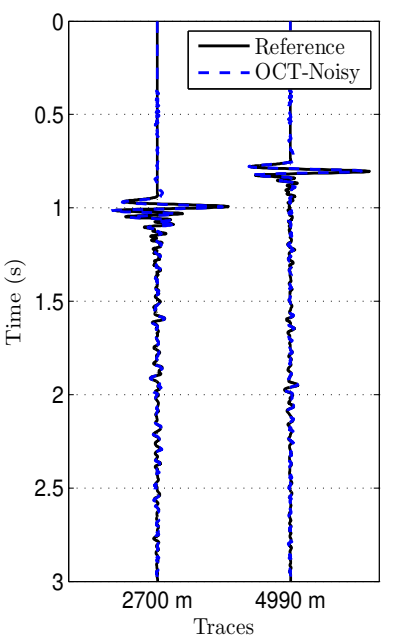

(d)

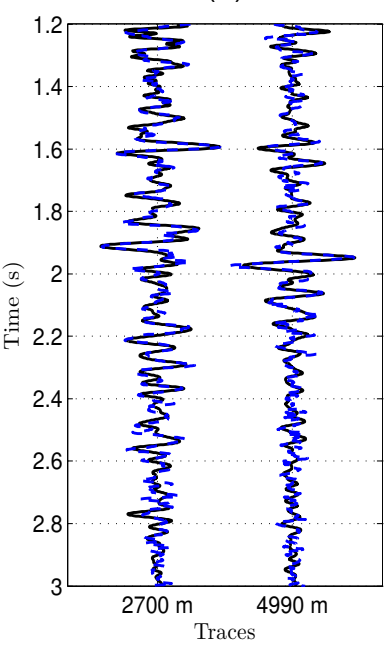

Figure 11. Traces comparison between Reference dataset and: (a) Original Noisy dataset; (b) Regularized Noisy (data after OCT method); (c) Zoom of (a); (d) Zoom of (b).

1 GPU Nvidia Tesla V100 16GB. Since the charging for most services follows the pay-as-you-go model, i.e., the users only pay how much they use for each virtual machine, thereby, the maximum iteration used as a stop criterion respects a use time (limiting expenses). Thus for the Noisy and Bit datasets, the maximum iteration analyzed was 110 , and for the other datasets, we reached the double, that is, 220 iterations.

We assume that the sea bottom is planar at depth $z=0.5 \mathrm{~km}$, and the water velocity is $1.5 \mathrm{~km} / \mathrm{s}$. In the inverse process, the same numerical scheme of finite difference is used. The initial velocity model for start iteration in the FWI method for all experiments is illustrated in Figure 17. This model was obtained by applying a smoothing filter, i.e., a central moving average applied in eighty-one points for each direction to the original Marmousi model.

To discuss the results obtained by the FWI method, we divide the dataset into two sets. The first consists of the (original) prestack data, i.e., Reference, Noisy

and Bit datasets. The second dataset consists of the regularized data obtained in the previous sections by the OCT method, which we will denote by OCT-Noisy, OCT-Bit-A, and OCT-Bit-B. In each situation, these are the data that in the FWI are commonly called observed data.

Using Reference dataset, the velocity model obtained by the FWI is shown in Figures 18 (a), and 18 (b), respectively for 110 and 220 iterations. These solutions represent the best estimates. Therefore, we assume them as Reference solutions for the purpose of comparisons in other cases. The following results are obtained using the Noisy and Bit datasets as input to the observed data in the FWI method, which are respectively shown in Figures 19 (a), and 19 (b). We note that the input data affects the velocity model update in these cases, as we use the same starting model and starting points in the inversion.

Furthermore, as expected, the Reference solution has better resolution. The rectangles indicate the regions that we will compare more carefully with the OCT method results. The different colors represent each aspect that can be found in a complex medium: the white rectangle shows a region with stratified layers, the red rectangle shows the most complex area (containing geological faults), and the green rectangle shows an area with a high contrast of velocity. Moreover, the estimated velocities are also compared to the true ones. However, we do not use any regularization/penalization strategies or filters that can help in the FWI final solution as multiscale strategies (Bunks et al., 1995).

The results presented so far only use the original prestack data. Thus, we can see that the model using the Noisy dataset has a slightly better resolution than the model estimated using the Bit dataset.

Now, we move to the second dataset, that is, the one that was regularized by the OCT method. Figures $20(a)$, and 20 (b) show the estimated model for the regularized Noisy dataset (OCT-Noisy) after 110 and 220 iterations, respectively. It is possible to observe from the white and green rectangles that we obtain more resolution than the original Noisy dataset. Nevertheless, in the red rectangle region at 110 iterations, the solution presents a poor resolution. However, with more iterations, the FWI method improved the estimation, which was possible since the observed data is less affected by noise. That is, the fit tends to be better.

The next solution was obtained using the OCT-Bit-A dataset as input for the FWI method. The estimated velocity model is shown in Figures 21 (a), and 21 (b) at 110 and 220 iterations, respectively. For both iterations, we obtain a velocity model with a good approximation to the Reference solution and a good improvement when compared to the highlighter areas in Figure 19 (b), particularly for the region given by the green and red rectangles. Finally, using regularized Bit dataset for the case B (OCT-Bit-B) for comparison, we have a slightly better solution than the solution of Case $A$, as we can see in Figures 22 (a) and 22 (b), at 110 and 220 itera- 
(a)

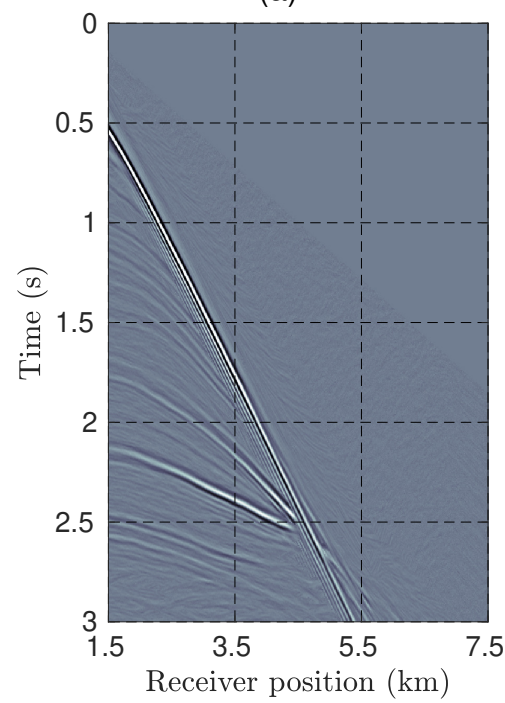

(b)

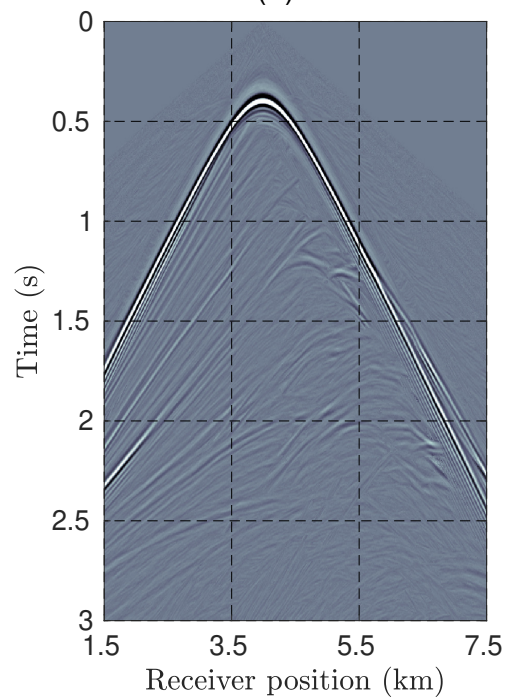

(c)

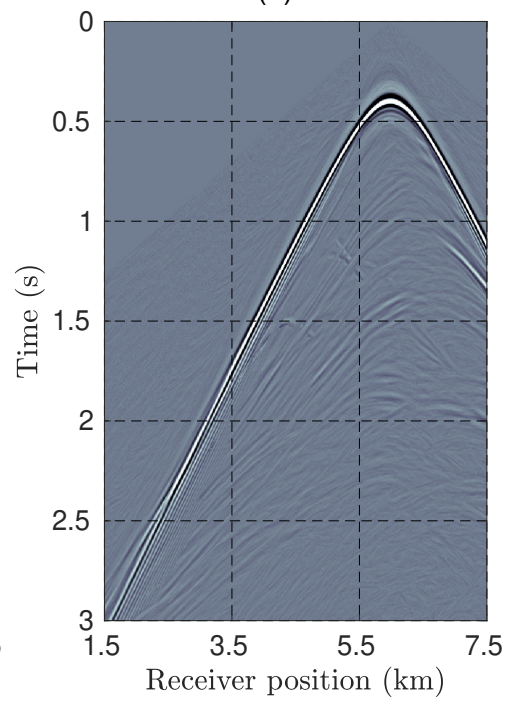

Figure 12. Bit dataset regularized (case A) by OCT using parameters from Noisy data for the shot position: (a) $1 \mathrm{~km}$; (b) $4 \mathrm{~km}$; (c) $6 \mathrm{~km}$.

(a)

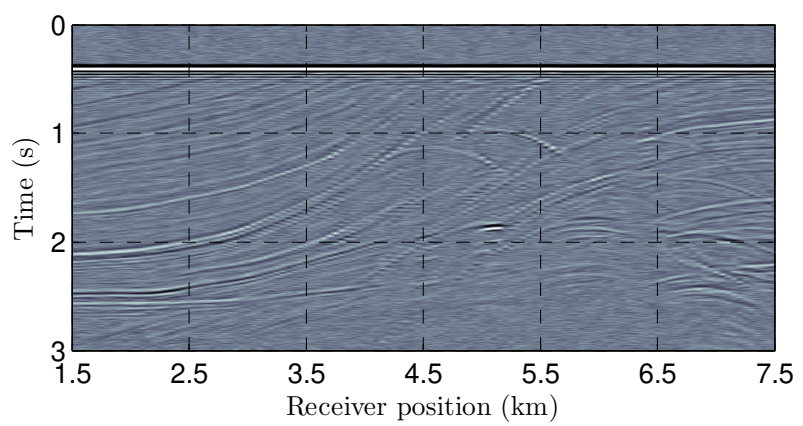

(b)

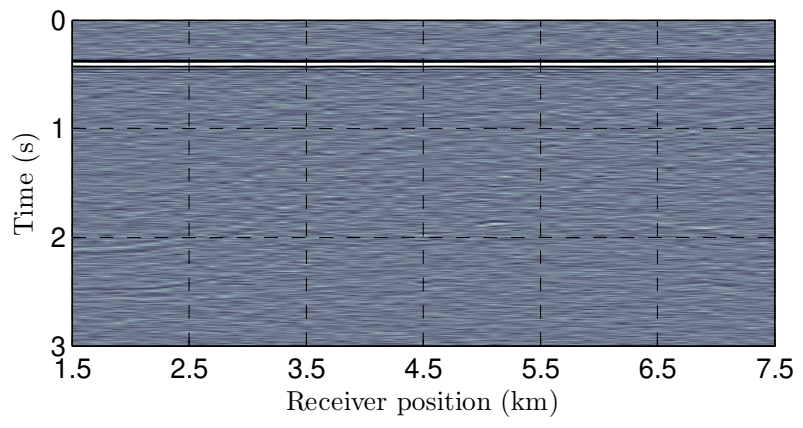

Figure 13. ZO section: (a) Bit dataset regularized (case A); (b) Original Bit dataset with noise.

tions, respectively. Although this case does not contain noise in the input data, the challenge faced was to estimate the OCT parameters from the prestack Bit dataset in order to increase the fold coverage about ten times. However, we have seen that the method works satisfactorily in the presence of noise, thus similar and even better solutions can be expected with greater expertise to obtain the parameters.

To show quantitative improvements using observed data after regularized by the OCT method, we use the following equation representing an absolute mean error between the true velocity model, $v_{i}$, and the estimated model $v_{i}^{k}$ in iteration $k$, that is,

$$
C\left(v^{k}\right)=\frac{1}{N} \sum_{i=1}^{N}\left(\left|\frac{v_{i}-v_{i}^{k}}{v_{i}}\right|\right),
$$

where the expression was normalized to the maximum value and $k=0.1 .2, \ldots, K$ are the $\mathrm{FWI}$ iterations for $K=110$ or $K=220$.

Equation (1) is a measure of prediction accuracy. Figure 23 shows the cost for all experiments performed; the Reference solution (black line) was the best solution, while the worst solutions are given by the original Noisy and Bit dataset (blue and red lines, respectively). After applying the OCT method we obtain intermediate solutions closer to the Reference solution, where the brown line represents the regularized Noisy data, the orange line represents regularized Bit data for Case $A$ and magenta line for Case $B$.

Another advantage of combining FWI and OCT methods is shown in Table 2, which compares CPU times (approximately) for running 220 iterations on the cloud. In other words, the shorter the execution time, the cheaper it becomes to use cloud computing. Moreover, we obtained CPU time closer to the Reference solution for regularized data by the OCT method, while for the non-regularized dataset, it took an average of 76 hours, which means that regularized data by the OCT method achieved a reduction of approximately ten times. The execution time for the regularization using the OCT method did not affect the final time shown in Table 2, since our algorithm is highly scalable and implemented in GPU.

Another benefit of combining the FWI solution using the observed data obtained by OCT regularization can be an alternative to decrease the operating cost of 
(a)

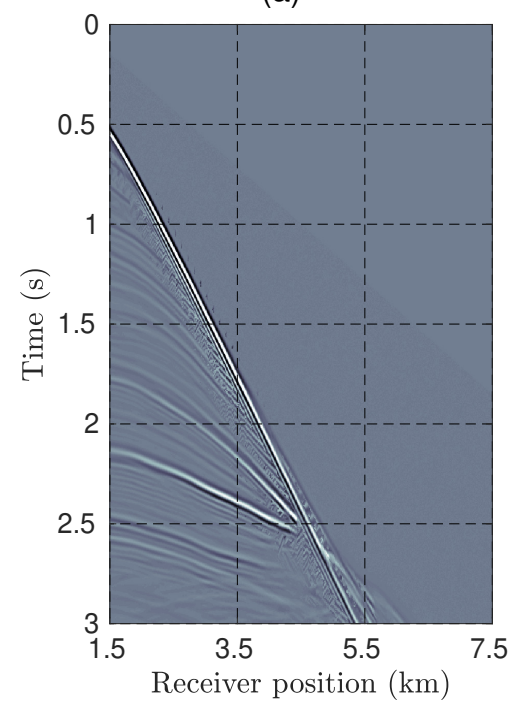

(b)

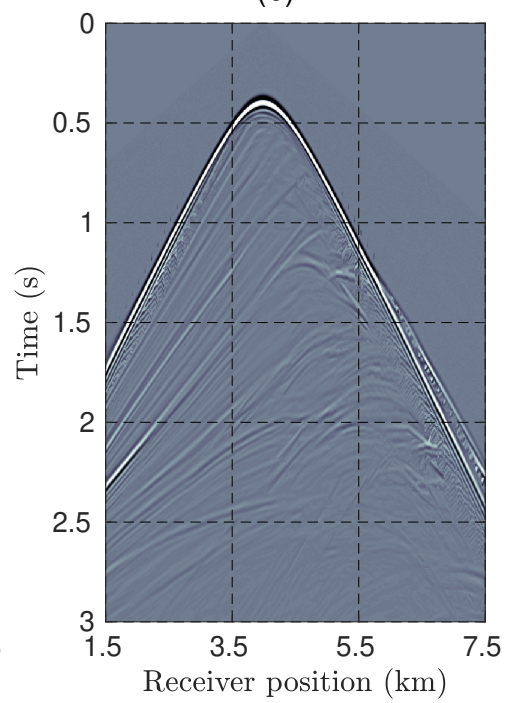

(c)

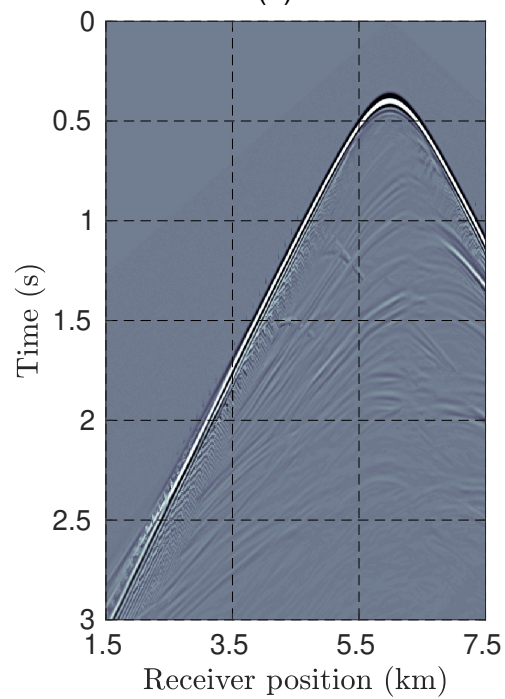

Figure 14. Bit dataset regularized (case B) by OCT using parameters from Bit data (noise free) for the shot position: (a) $1 \mathrm{~km}$; (b) $4 \mathrm{~km}$; (c) $6 \mathrm{~km}$.

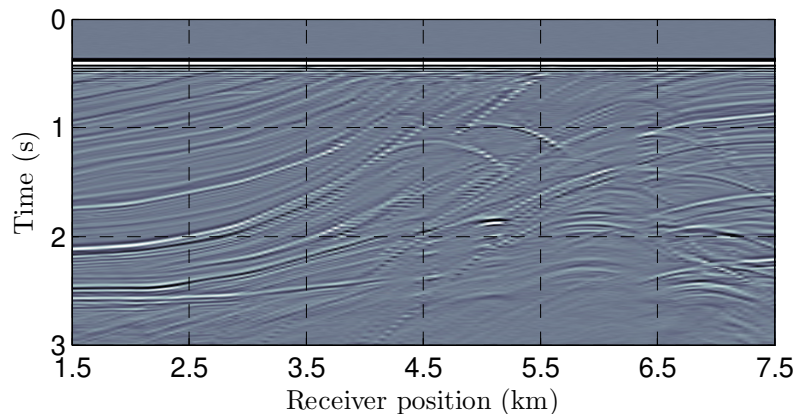

Figure 15. Zero-offset section of the Bit dataset regularized (case B).

Table 2. : Approximate CPU execution time running 220 iterations for FWI (in hours).

\begin{tabular}{|l|c|c|c|}
\hline Original datasets & Time & $\begin{array}{c}\text { Regularized } \\
\text { data }\end{array}$ & Time \\
\hline Reference & 6.0 & OCT-Noisy & 7.0 \\
\hline Noisy & 78.0 & OCT-Bit-A & 7.2 \\
\hline Bit & 74.0 & OCT-Bit-B & 7.0 \\
\hline
\end{tabular}

an OBN acquisition, since the obtained solutions have presented a similar quality in relation to the reference model. Also, this approach has shown improvements with respect to the signal-to-noise enhancement, which contributes to a suitable inversion procedure.

\section{CONCLUSIONS}

In this paper, we have shown an alternative to reduce the cost of an OBN acquisition in non-deeper water. However, the results obtained are not only restricted to this type of acquisition. The OCT regularization tech- nique can improve the illumination of the reflectors, which can sometimes be a problem simply because they may not be positioned in some areas of interest. Besides that, the regularization of data by the OCT method proved to be a suitable assistant of seismic imaging methods, particularly for the FWI method, resulting in a faster convergence due to the suitable treatment of noise and increasing fold coverage obtained by the OCT method. The inversion solution is very similar to the solution of a reference dataset solution model. There are no significant losses, but in some regions, we have even improved the quality of the solution. Based on our experiments, in future works, the presented solutions can be improved by applying a meticulous analysis of OCT parameters and adopting a multi-scale strategy on the FWI method and the application on real seismic datasets.

\section{ACKNOWLEDGMENTS}

This work was possible thanks to the support of Petrobras and Fapesp/Cepid \#2013/08293-7-Brazil. The authors also thank the High-Performance Geophysics (HPG) team for technical support. 
(a)

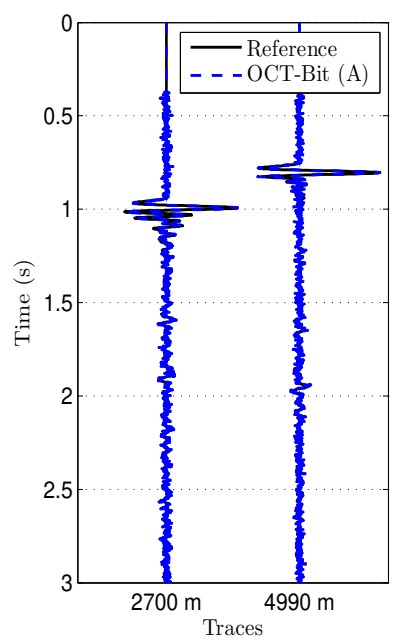

(c)

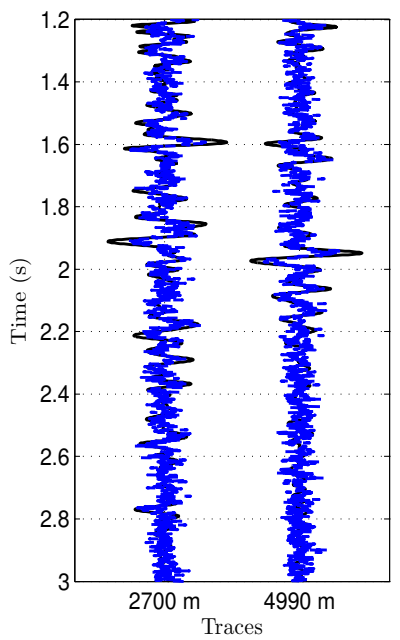

(b)

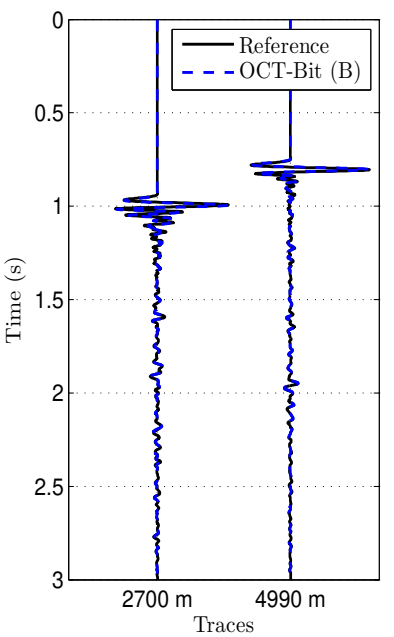

(d)

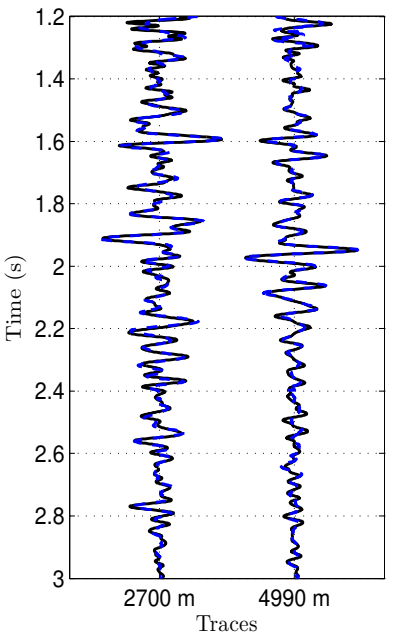

Figure 16. Comparison between traces of the Reference dataset (black line) with Regularized Bit dataset (blue line) for: (a) Case A ; (b) Case B; (c) Zoom of (a); (d) Zoom of (b).

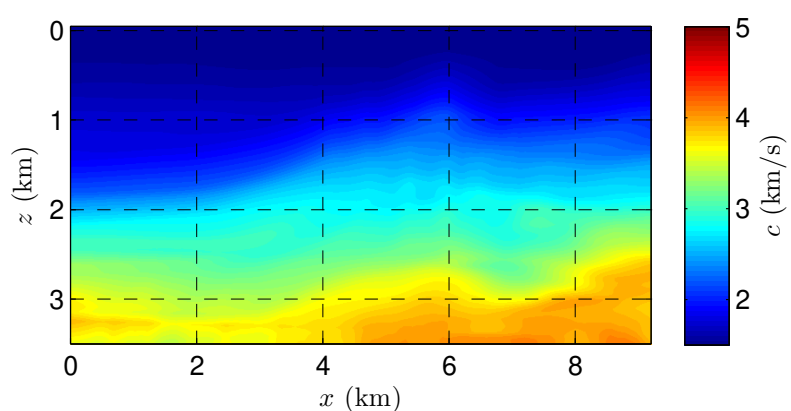

Figure 17. Initial approximation for the velocity model used in FWI method .

(a)

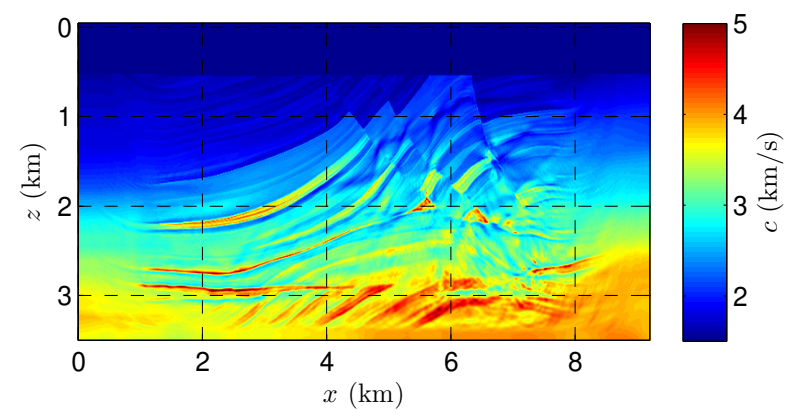

(b)

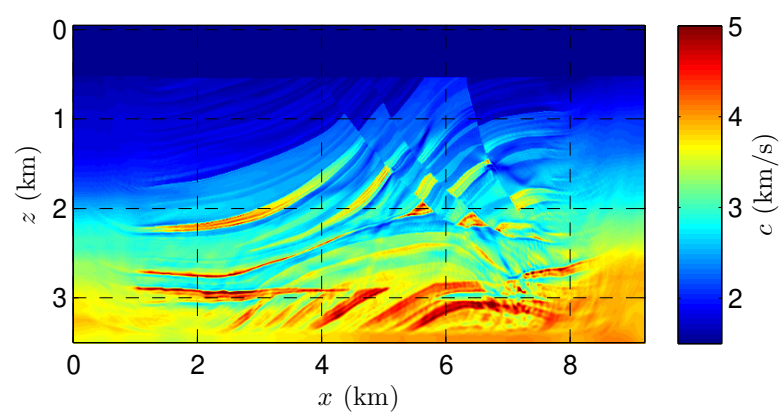

Figure 18. Estimated velocity model using Reference dataset after: (a) 110 iterations; (b) 220 iterations.

(a)

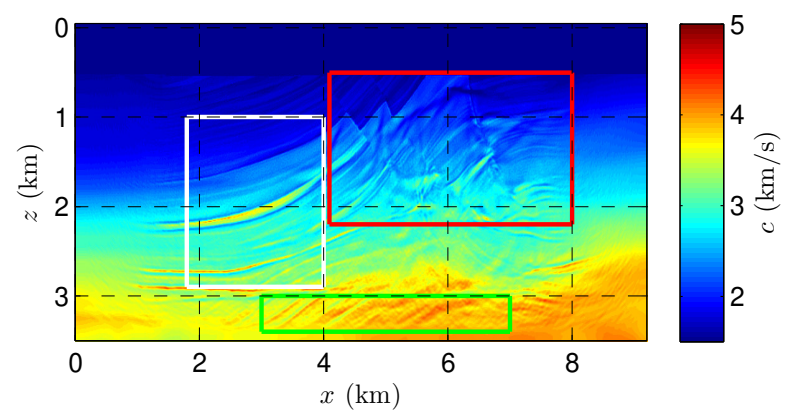

(b)

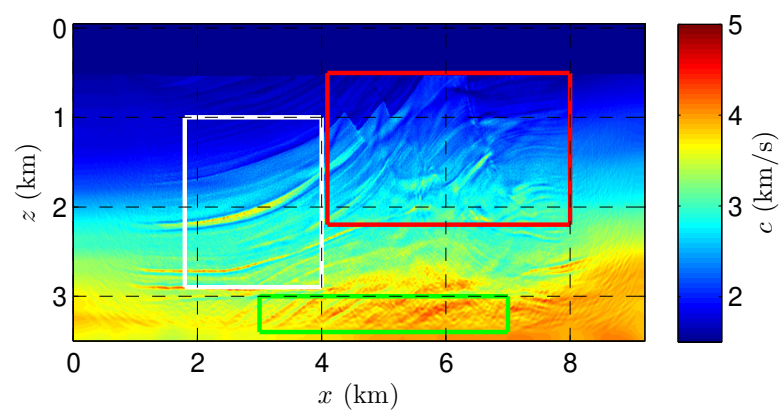

Figure 19. Estimated velocity model after 110 iterations using non-regularized dataset: (a) Noisy; (b) Bit. 
(a)

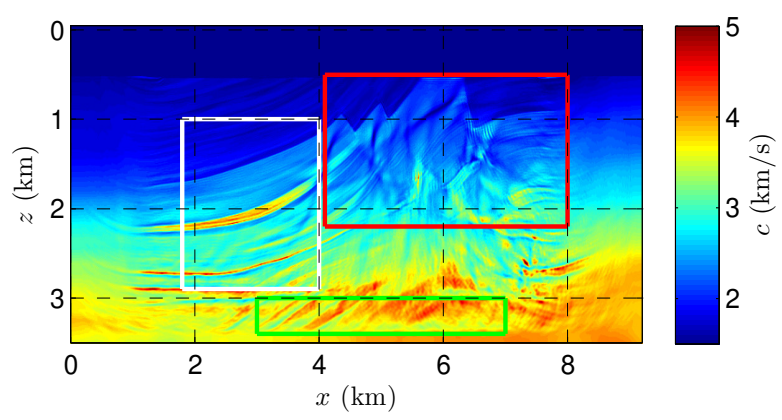

(b)

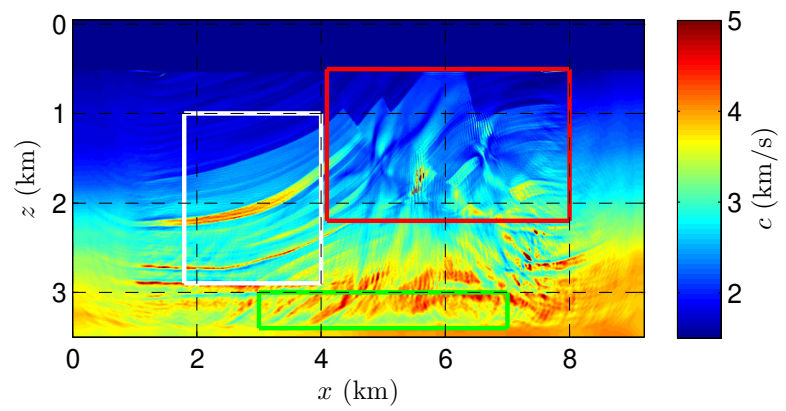

Figure 20. Estimated velocity model using regularized Noisy dataset after: (a) 110 iterations; (b) 220 iterations.

(a)

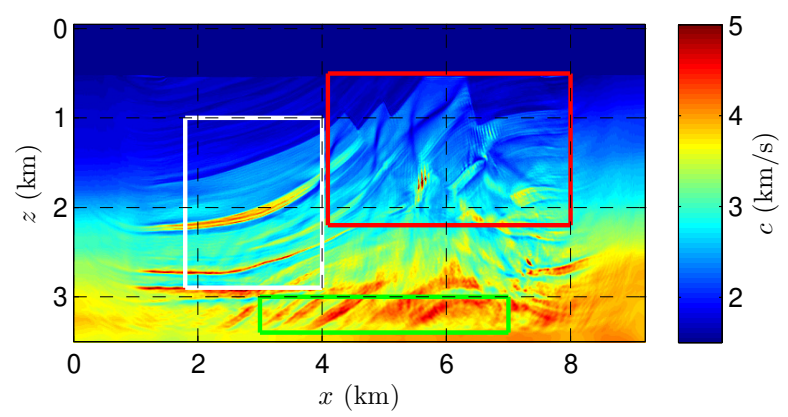

(b)

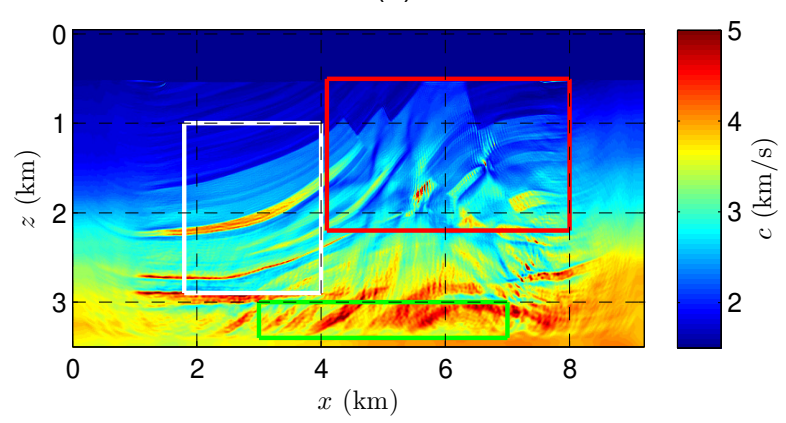

Figure 21 . Estimated velocity model using regularized Bit dataset case A after: (a) 110 iterations; (b) 220 iterations. (a)

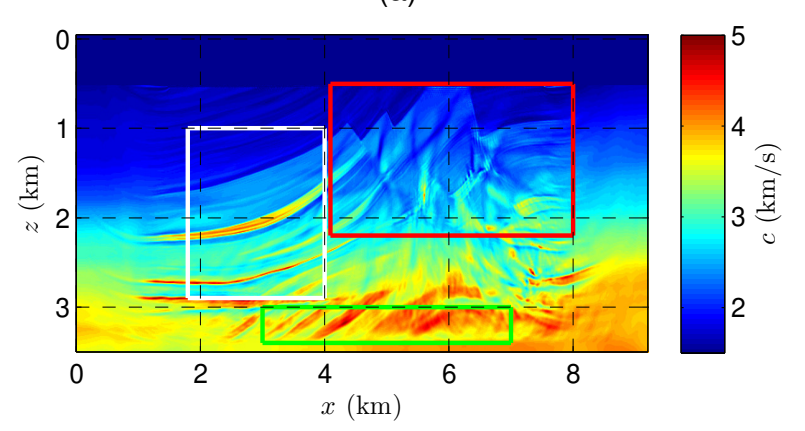

(b)

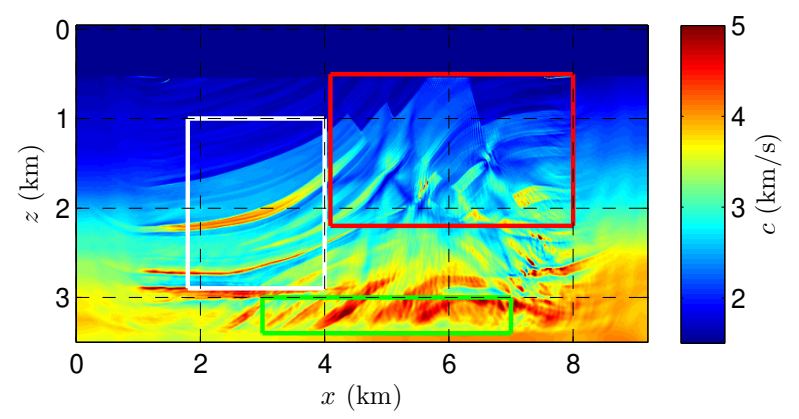

Figure 22. Estimated velocity model using regularized Bit dataset case B after: (a) 110 iterations; (b) 220 iterations.

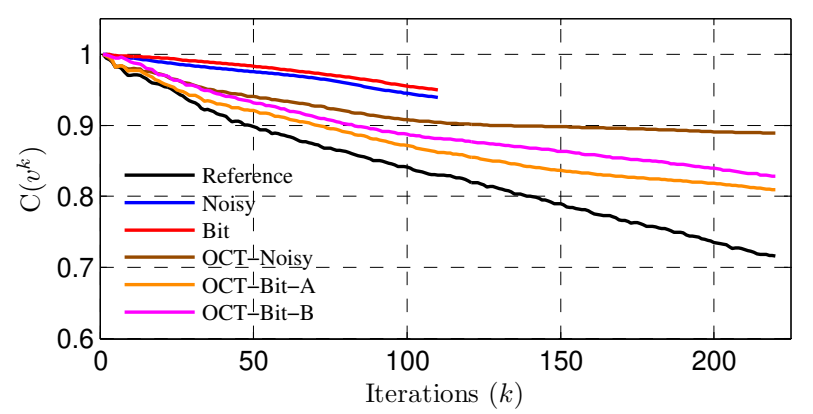

Figure 23. Cost analysis per iterations for FWI using mean absolute error. 


\section{REFERENCES}

Abreo, S.; Ramirez, A.; Vivas, F.; Gonzalez, H.; Wiarda, E.; Martinez, R.; Serrano, J.O.; Gonzalez, C. Diving-wave FWI methodology applied to a Colombian Caribbean data set. The Leading Edge 2018, 37, 242-320.

Alford, R.M.; Kelly, K.R.; Boore, D.M. Accuracy of finitedifference modeling of the acoustic wave equation. Geophysics 1974, 39, 834-842.

Asnaashari, A.; Brossier, R.; Garambois, S.; Audebert, F.; Thore, P.; Virieux, J. Regularized seismic full waveform inversion with prior model information. Geophysics 2013, 78, R25-R36.

Bleistein, N.; Cohen, J.K.; Jr., J.W.S. Mathematics of multidimensional seismic imaging, migration and inversion; Vol. 13, Interdisciplinary Applied Mathematics, Springer, New York, NY. 510 pp, 2001.

Bleistein, N. Mathematical methods for wave phenomena; Computer Science and Applied Mathematics, Academic Press INC., Orlando, FL, 341 pp, 1984.

Bunks, C.; Saleck, F.M.; Zaleski, S.; Chavent, G. Multiscale seismic waveform inversion. Geophysics 1995, 60, 1457-1473.

Carcione, J.M.; Herman, G.C.; Krood, A.P.E. Seismic modeling. Geophysics 2002, 67, 1304-1325.

Coimbra, T.A.; Novais, A.; Schleicher, J. Offsetcontinuation (OCO) ray tracing using OCO trajectories. Studia Geophysica et Geodaetica 2012, 56, 6582.

Coimbra, T.A.; Novais, A.; Schleicher, J. Offsetcontinuation stacking: Theory and proof of concept. Geophysics 2016, 81, V387-V401.

Crase, E.; Pica, A.; Noble, M.; McDonald, J.; Tarantola, A. Robust elastic nonlinear waveform inversion: Application to real data. Geophysics 1990, 55, 527-538.

Deregowski, S.M.; Rocca, F. Geometrical optics and wave theory of constant offset sections in layered media. Geophysical Prospecting 1981, 29, 374-406.

Fichtner, A. Full seismic waveform modeling and inversion; Advances in Geophysical and Environmental Mechanics and Mathematics, Springer-Verlag, Berlin, Heidelberg, 352 pp, 2011.

Fomel, S. Theory of differential offset continuation. Geophysics 2003, 68, 718-732.

Fomel, S. Shaping regularization in geophysicalestimation problems. Geophysics 2007, 72, 1MAZ35.

Forel, D.; Benz, T.; Pennington, W. Seismic data processing with Seismic Un ${ }^{*}$ : A $2 D$ seismic data processing primer; Course notes series n.12, Society of Exploration Geophysicists, 291 pp, 2005.

Gaiser, J. 3C Seismic and VSP: Converted waves and vector wavefield applications; Distinguished Instructor Series, No 2, Society of Exploration Geophysicists, Tulsa, OK, 636 pp, 2016.

Gülünay, N. Seismic trace interpolation in the Fourier transform domain. Geophysics 2002, 68, 355-369.

Holberg, O. Computational aspects of the choice of the operator and sampling interval for numerical differentiation in large-scale simulation of the wave phenomena. Geophysical Prospecting 1987, 35, 625-655.

Huang, R.; Zhang, Z.; Wu, Z.; Wei, Z.; Wang, P. Full-waveform inversion for full-wavefield imaging: Decades in the making. The Leading Edge 2021, 40, 314-392.

Husted, B.; Operto, S.; Virieux, J. Mixed-grid staggeredgrid finite difference methods for frequency-domain acoustic wave modelling. Geophysical Journal International 2004, 157, 1269-1296.

Jäger, R.; Mann, J.; Höcht, G.; Hubral, P. Commonreflection-surface stack: Image and attributes. Geophysics 2001, 66, 14-357.

Jouno, F.; Martinez, A.; Ferreira, D.; Donno, D.; Khalil, A. Potential and challenges of FWI with OBN data to image the pre-salt of the Santos Basin. 16th International Congress of the Brazilian Geophysical Society, Rio de Janeiro, Brazil, 2019, pp. 1-5.

Kelly, K.R.; Ward, R.W.; Treitel, S.; Alford, R.M. Synthetic seismograms: A finite-difference approach. Geophysics 1976, 41, 2-27.

Kosloff, R.; Kosloff, D. Absorbing boundaries for wave propagation problems. Journal of Computational Physics 1986, 63, 363-373.

Lailly, $P$. The seismic inversion problem as a sequence of before stack migrations. Conference on Inverse Scattering: Theory and Application, eds. Bednar J.B., Robinson E., Weglein A. Society for Industrial and Applied Mathematics, 1983, Vol. 1, Proceedings in Applied Mathematics Series, pp. 206-220.

Leeuwen, T.V.; Herrmann, F.J. A penalty method for PDE-constrained optimization in inverse problems. Inverse Problems 2016, 32, 1-27.

Liu, D.C.; Nocedal, J. On the limited memory BFGS method for large scale optimization. Mathematical Programming 1989, 45, 503-528.

Nocedal, J.; Wright, S.W. Numerical optimization; Springer Series in Operations Research, SpringerVerlag, New York, NY, 664 pp, 1999.

Plessix, R.E. A review of the adjoint state method for computing the gradient of a functional with geophysical applications. Geophysical Journal International 2006, 167, 495-503.

Prieux, V.; Lambaré, G.; Operto, S.; Virieux, J. Building starting models for full waveform inversion from wide-aperture data by stereotomography. Geophysical Prospecting 2012, 209, 1629-1647.

Qu, S.; Verschurr, E.; Chen, Y. Full-waveform inversion and joint migration inversion with automatic directional total variant constraint. Geophysics 2019, 84, 1MA-Z11.

Sommerfeld, A. Partial Differential equations in Physics; Vol. 1, Academic Press INC., New York, NY, 334 pp, 1949.

Souza, A.A.V.B.; Schleicher, J. Least squares imaging using $\ell l_{1}$ and student residual. 15th International Congress of the Brazilian Geophysical Society, Rio de Janeiro, Brazil, 2017, pp. 1-5. 
Tarantola, A. Inversion of seismic reflection data in The acoustic approximation. Geophysics 1984, 49, 12591266.

Trad, D.O.; Ulrych, T.J.; Sacchi, M.D. Accurate interpolation with high-resolution time-variant Radon transforms. Geophysics 2002, 67, 348-663.

Versteeg, R. The Marmousi experience: Velocity model determination on a synthetic complex data set. The Leading Edge 1994, 13, 927-936.

Virieux, J.; Operto, S. An overview of full-waveform inversion in exploration geophysics. Geophysics 2009, 74, 127-152.

Wang, C.; Yingst, D.; Bai, J.; Leveille, J.; Farmer, P.; Brittan, J. Waveform inversion including well constraints, anisotropy and attenuation. The Leading Edge 2013, 32, 1005-1176.

Wang, D.; Chen, C.; Zhuang, D.; Mei, J.; Wang, P. Land FWI: Challenges and Possibilities. 82nd EAGE Annual Conference \& Exhibition, Jul 2020, 2020, pp. 15.

Wellington, P.; Brossier, R.; Virieux, J. Preconditioning full-waveform inversion with efficient local correlation operators. Geophysics 2019, 84, ix-Z16.

Wu, S.; Wang, Y.; Zhang, Y.; Chang, X. Limited-memory BFGS based least-squares pre-stack Kirchhoff depth migration. Geophysical Journal International 2015, 202, 738-747.

A.C.: implemented the seismic inversion and performed all the experiments and discussed the results. J.R.: was responsible for implementing the GPU framework.T.A.C.: was the work supervisor and discussed the structure of numerical experiments performed in this paper. G.B.: performed the experiments to search for the optimal parameters. M.T.: discussed and suggested improvements throughout the work. All author have read and revised the work and agree to the published of paper.

Received on September 3,2019/ Accepted em October 28, 2021. 